\title{
Information transmission in spectral color naming'
}

\author{
ROBERT T. KINTZ, ${ }^{2}$ JOHN A. PARKER, ${ }^{3}$ AND ROBERT M. BOYNTON \\ UNIVERSITY OF ROCHESTER
}

The concepts of information theory are applied in an analysis of color naming data where various numbers of response categories are permitted for the description of 21 spectral stimuli. The results are summarized by en empirical

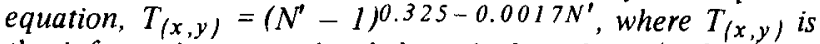
the information transmitted through the subject (in bits) and $N^{*}$ is the number of response categories used by the $S$. This function has a maximum value close to 2.6 bits. It is suggested that 28 response categories, corresponding to seven steps between adjacent hues of the color circle, constitute a good choice for experiments of this type.

A number of recent studies have been concerned with how perceived hue varies with the wavelength of light (Dimmick \& Hubbard, 1939; Thomson, 1954; Jameson \& Hurvich, 1959; Beare, 1963; Boynton, Schafer, \& Neun, 1964; Boynton \& Gordon, 1965; Sternheim \& Boynton, 1966; Beare \& Siegel, 1967). Although this relation has often been described in a qualitative way since Newton first declared that the spectrum contained seven bands of color, efforts to quantify it are rather recent. These have utilized a variety of experimental procedures and methods of data analysis.

In our laboratory, we have used mainly a method that requires the $S$ to limit his use of color names to four, and to make either one or two responses to each of a series of randomly-ordered, equally-bright flashes of monochromatic lights. The names that have been used are red, yellow, green, and blue (RYGB), following Hering's conception (see Hering, translated by Hurvich \& Jameson, 1964) that all bright colors can be adequately described as combinations of these four, none of which contains any trace of the other three. (In some experiments we have also permitted the use of "white" judgments, but for simplicity these are not to be discussed here, nor were they used in the experiment to be described.) The $S$ is told that if the hue of the light appears to be unique, he should give only one response. Most of the time two hue components are seen; in these cases we permit the $S$ to make two responses. The first is to indicate the dominant hue perceived, the second to denote the secondary (weaker) hue component. We have scored responses by arbitrarily assigning a point value of three to each response. When a unique response is given, the entire value is assigned to the appropriate response category. When double responses are given, points are split on a two-to-one basis, with the heavier weight assigned to the category of the dominant component.

A problem to which we address ourselves in this paper is whether the 12 response categories of this method (BR, B, BG, $G B, G, G Y, Y G, Y, Y R, R Y, R$, and $R B$ ) allow the $S$ to transmit as much information as possible about the stimulus. An example will indicate why 12 categories may be inadequate: if the $S$ sees a greenish blue of some kind, he is not by our previous method permitted to communicate how much green is seen relative to the blue. No matter how it may look to him, we arbitrarily weight the green response as if the secondary green component had appeared half as strong as the blue. But, in fact, the S may have seen the green as very nearly as strong as the blue, or, at the other extreme, the flash may have been just barely discriminable from unique blue.

These observations have led us to suspect that the use of more response categories might permit the $S$ to transmit more information. For example, some $S$ s show a more or less flat region in the green-blue part of the spectrum where wavelength discrimination is poor. Here the green function is constrained to assume a value near $67 \%$, with the blue function taking up the remaining $33 \%$. This is the result of our limitation of response categories as reflected in the scoring system; almost all of the individual responses are "Green-Blue" over this flat range. Some Ss have volunteered the opinion that they could have made finer discriminations had they been permitted to do so. We did in fact perform one study (Sternheim \& Boynton, 1966) where response strengths were judged on a nearly continuous scale, from 0 to 100 . The results suggest, but do not prove, that more information per trial might be obtained using this more continuous response procedure.

The experiment to be described was therefore designed to determine how many bits of information (Shannon, 1948) are transmitted through a $S$ as a function of the number of response categories permitted. An increase in the number of categories could have been accomplished by increasing the number of hue names, increasing the number of discrimination steps allowed in judging a secondary hue component, or both. The results of Sternheim and Boynton suggest that the addition of further hue categories would not improve the result. Also, we wanted to increase the number of response categories to more than 40 , which is clearly impossible just by adding more color names. Therefore, we elected to use the second method, keeping the number of names at four and allowing finer discrimination of relative secondary response strength.

The data of a color naming experiment can be put into a matrix having the general form shown in Table 1 . Some cells in the matrix contain a frequency representing the number of times $\left[\left(n_{i j}\right) \neq 0\right]$ that a given color name was assigned to a particular stimulus. Using the standard procedures originally developed by Shannon, we can compute the following values, each in bits:

(1) Information in the stimulus:

$$
H_{(x)}=\log _{2} n-(1 / n) \stackrel{M}{\Sigma} n_{i} \log _{2} n_{i}
$$

(2) Information in the response:

$$
H_{(y)}=\log _{2} n-(1 / n) \stackrel{N}{\Sigma} n_{j} \log _{2} n_{j}
$$

(3) Information occurring jointly in stimulus and response:

$$
H_{(x, y)}=\log _{2} n-(1 / n) \stackrel{M N}{\Sigma} n_{i j} \log _{2} n_{i j}
$$

(4) Information transmitted through the subject:

$$
T_{(x, y)}=H_{(x)}+\dot{H}_{(y)}-H_{(x, y)}
$$

In terms of the matrix of Table 1, note that complete transmission of information can occur only if the number of response categories is equal to or greater than the number of stimulus categories. In our earlier work, more than 20 stimulus categories were used, but only 12 response categories were permitted; perfect information transmission could not therefore have occurred. But maximum transmission could 
Table 1

Matrix in which Data Relating Color Naming Responses to Spectral Stimuli are ordered in Order to Permit Informational Analysis. Entries are Frequencies of Occurrence given Repeated Presentation of the Stimuli

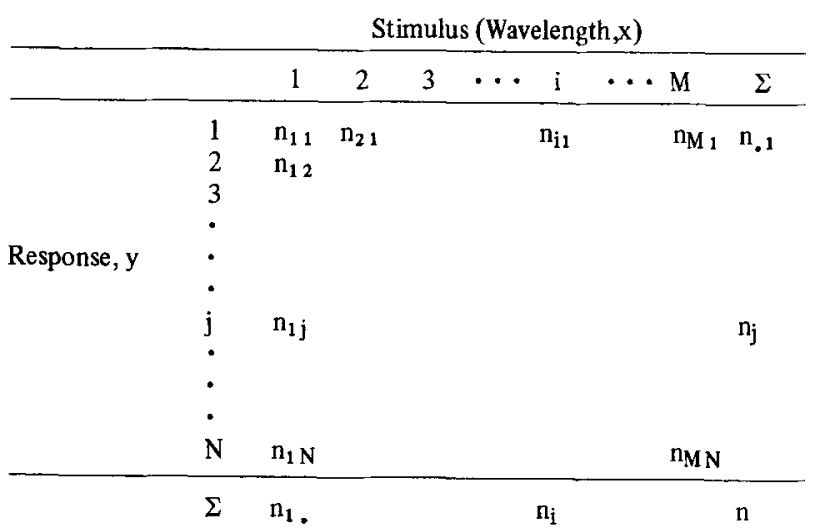

have, since increasing the number of response categories does not necessarily increase the amount of information transmitted.

\section{METHOD AND PROCEDURE}

The stimuli used were the following 21 spectral wavelengths: $450,461,470,478,486,492,502,511,522$, $533,546,555,566,575,589,592,600,609,620,629$, and $640 \mathrm{~nm} .{ }^{4}$ These were approximately equated for brightness by being presented at 1000 times the threshold luminance for each $\mathrm{S}$ as established by prior experiment. Stimuli were $3 \mathrm{deg}$ in diam, centrally-presented in Maxwellian view, with wavelength controlled by the position of an interference wedge located at a source image. They were flashed for $250 \mathrm{msec}$, self-delivered by the $\mathrm{S}$. Each $\mathrm{S}$ received each stimulus 10 times in random order of presentation for each of four experimental conditions to be outlined below.

\section{Experimental Conditions}

Condition 44. In this condition, 44 response categories were available. Those in the spectral range from unique blue to unique green are given in the third column of Fig. 1. Analogous responses were permitted to cover the remaining three quadrants of the full color circle. The $S$ was instructed to consider that the primary response had a strength of 6 , so that (for example) a response of "BG5" indicated a greenish-blue that was very nearly ( $5 / 6$ ths $)$ as green as blue.

Condition 24. In this condition, 24 response categories were used. (See Column 4 of Fig. 1.) The primary component in this case had a value of 3 ; the response $B / G$ could also have been written $\mathrm{BG} 3$ or GB3 because it is used to indicate that the two components are perceived as so nearly equal that to weight them in a ratio of $2: 3$ or $3: 2$ would be a less accurate description.

Condition 12. This was the standard 12-category response procedure previously used in the study by Boynton and Gordon, and is represented by the second column of Fig. 1.

Condition 8. This was an eight-category condition illustrated in the extreme right-hand column of Fig. 1.

The four conditions were explored in separate sessions, in the order that they have been listed here, excepting that not all conditions were employed for each $\mathrm{S}$.

Derived conditions. Fig. 1 shows how it is possible, by lumping response categories together in certain ways, to collapse the data of the 44-category condition into the categories of the other three experimental conditions. This was done in order to compare the results with those obtained experimentally. In addition, 16- and 4-category conditions were derived as shown which were not tested experimentally.

\section{Subjects}

Two of the authors served alternately as S and E. Both RTK and JP observed under Condition 44, which permitted analysis for all derived conditions for both Ss. S JP served under Conditions 8 and 24, and RTK served under Condition 12.

\section{RESULTS}

In Fig. 2, information transmitted is plotted as a function of the number of response categories used. Experimental data are shown as the solid symbols; values deduced from the results for larger numbers of categories are plotted as open symbols. For the 44-category condition, one of the Ss transmits about 2.7 bits, the other 2.5 ; the use of fewer categories allows the transmission of less information.

Results for the two Ss for the 44-category condition (the triangles at the far right in Fig. 2) seem similar enough to allow a joint description of the data for the two Ss. With a total of only five experimental values, many functions, including even a linear one, could be drawn through the points. For this reason it is important to consider limiting cases beyond the bounds of the experimental values, so that extrapolations of the fitted function seem reasonable.

The function chosen, and drawn through the data, is an exponential:

$$
T_{(x, y)}=\left(N^{\prime}-1\right)^{0.325-0.0017 N^{\prime}}
$$

Here it will be recalled that $T_{(x, y)}$ is the amount of information transmitted; from here on we will use $\mathrm{N}^{\prime}$ for the number of response categories used. The derivation of this equation is based upon the following considerations. The expression $\left(N^{\prime}-1\right)$ must be used instead of $N^{\prime}$ in order for the equation to be meaningful when extrapolated to small values. When $N^{\prime}=2, T_{(x, y)}=1$, according to Eq. 5. This implies that exactly one bit of information is transmitted when only two response categories are permitted. This is an optimal result, but not an impossible one. When $\mathrm{N}^{\prime}$ becomes unity, there is only one response category and therefore no information can be transmitted. Equation 5 accounts for this satisfactorily.

Simple exponents of $\left(\mathrm{N}^{*}-1\right)$ were tried first, but no constant one proved capable of fitting the data points satisfactorily. For small values of $\mathrm{N}^{\prime}$, a relatively large exponent is needed, but this overpredicts the amount of

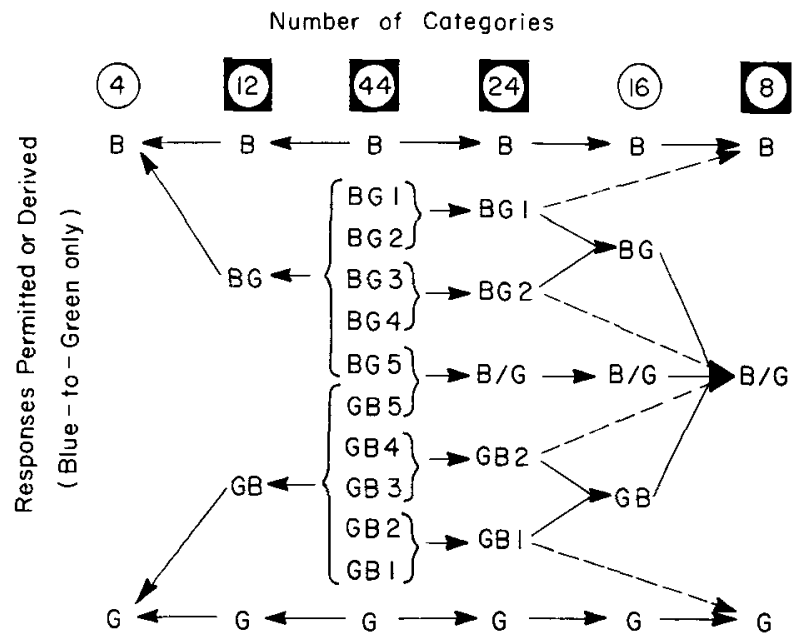

Fig. 1. Response categories used for one quadrant of the hue circle. The four experimental conditions actually used are indicated by the black squares. Responses for the 44-category condition were also analyzed by lumping them into fewer categories as indicated by the brackets and arrows. This analysis produced three categories for which experimental data were also available $(8,12$, and 24$)$ and two for which they were not (4 and 16). 


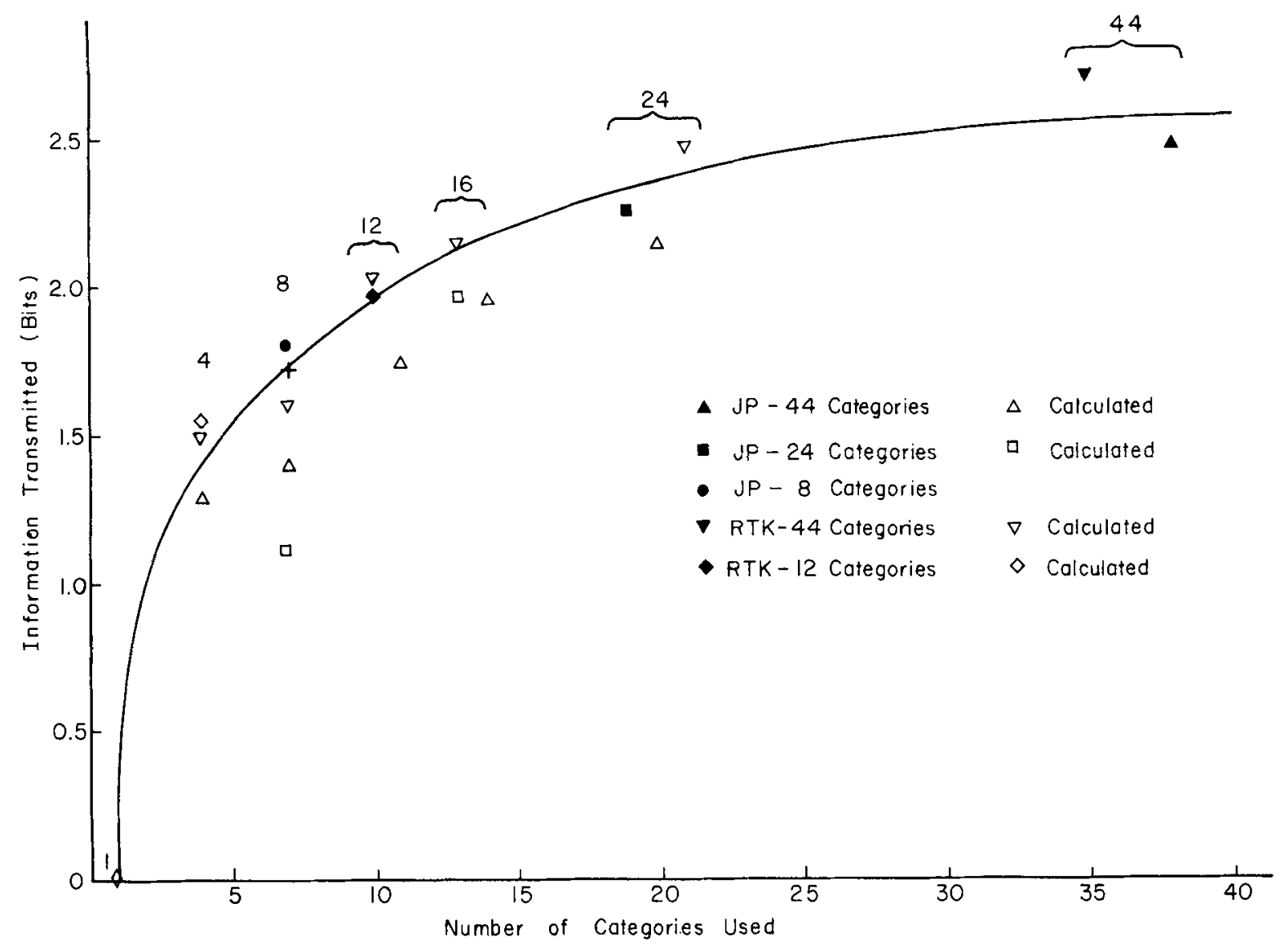

Fig. 2. Information transmitted through the $S$ as a function of the number of response categories used. Solid points are experimentally determined; open symbols refer to computations based upon data obtained with the larger number of categories shown in the inset. Numbers at the top show the number of response categories available to be used.

information transmitted under the 44-category condition. Conversely, larger values of $\mathrm{N}^{\prime}$ require a smaller exponent to fit the data well, but this leads to an underprediction of the amount of information transmitted for the 8-and 12-category conditions. Further, the use of any constant exponent implies that information transfer will increase indefinitely with increasing numbers of response categories. This leads to the absurd prediction that, somewhere between 150 and 200 categories (depending upon the exact choice of exponent) of information transmitted will exceed the information contained in the stimulus.

Other considerations lead us to believe that the function of Fig. 2. should reach a maximum, or approach a maximum asymptotically, and that the maximum probably has been reached for the 44-category condition. Sternheim and Boynton found-and others have noted the same phenomenon-that although Ss were allowed to use a 100-point scale, most of them rounded to the nearest five and, therefore, actually used at most a 20-point scale. Aithough our data do show clearly that there is an increase in transmitted information between the 24- and 44-category conditions, it should be realized that the Ss had only 11 steps to judge, not 44 , between adjacent unique hues.

The second term in the exponent of Eq. 5 is a correction factor that scales the exponent from larger toward smaller values with an increase in the number of categories. This produces a very flat maximum (2.56 bits) at $\mathrm{N}^{\prime}=41.45$. To within less than one part in a thousand, the curve has reached its maximum at $\mathrm{N}^{\prime}=40$ and maintains a constant value within $2 \%$ to $\mathrm{N}^{\prime}=50$. The extreme right-hand portion of the curve in Fig. 2 may therefore be taken as a maximum value over the working range.
It is not likely that categories in excess of 50 have much meaning in an experiment like this, so that the working range of the equation should be taken as from $N^{\prime}=1$ to $N^{\prime}=50$. If extrapolated beyond this range, the curve inflects and decreases very slowly, reaching $T_{(x, y)}=1$ bit at 191.1 categories, and zero at 415.8 . Since the equation should be regarded as an empirical one, no theoretical meaning should be attached to these extrapolated values.

\section{DISCUSSION}

\section{Comparison of Empirical and Calculated Values}

The calculated points (open symbols in Fig. 2) fall reasonably close to the experimental ones (closed symbols), but there are some sizeable discrepancies, the largest of these always placing the calculated points too low. This implies that after-the-fact collapsing of data into categories by the $E$ was not always done as efficiently as when the $\mathrm{S}$ did it for himself. The worst discrepancy is for JP when his data for the 24-category condition are collapsed into eight. Reference to the solid arrows in Fig. 1 will show how this was done. After the fact, it seemed that too many responses $(B G 1, B G 2, B / G$, $\mathrm{GB} 2$, and GB1) had been collapsed into the $\mathrm{B} / \mathrm{G}$ category, and that the agreement probably would have been better had $B G I$ been assigned to $B$ and $B G 2$ to $B / G$ (see dotted arrows in Fig. 1), with analogous changes elsewhere. The predictive failure as calculated implies that the use of the unique response categories will depend upon how many other categories are available, in agreement with conclusions previously reached by Sternheim and Boynton and by Beare and Siegel (1967). A recalculation of this value, using the procedure indicated by the dotted arrows, raised the aberrant point nearly to the empirical one (see "f" symbol in Fig. 2). 


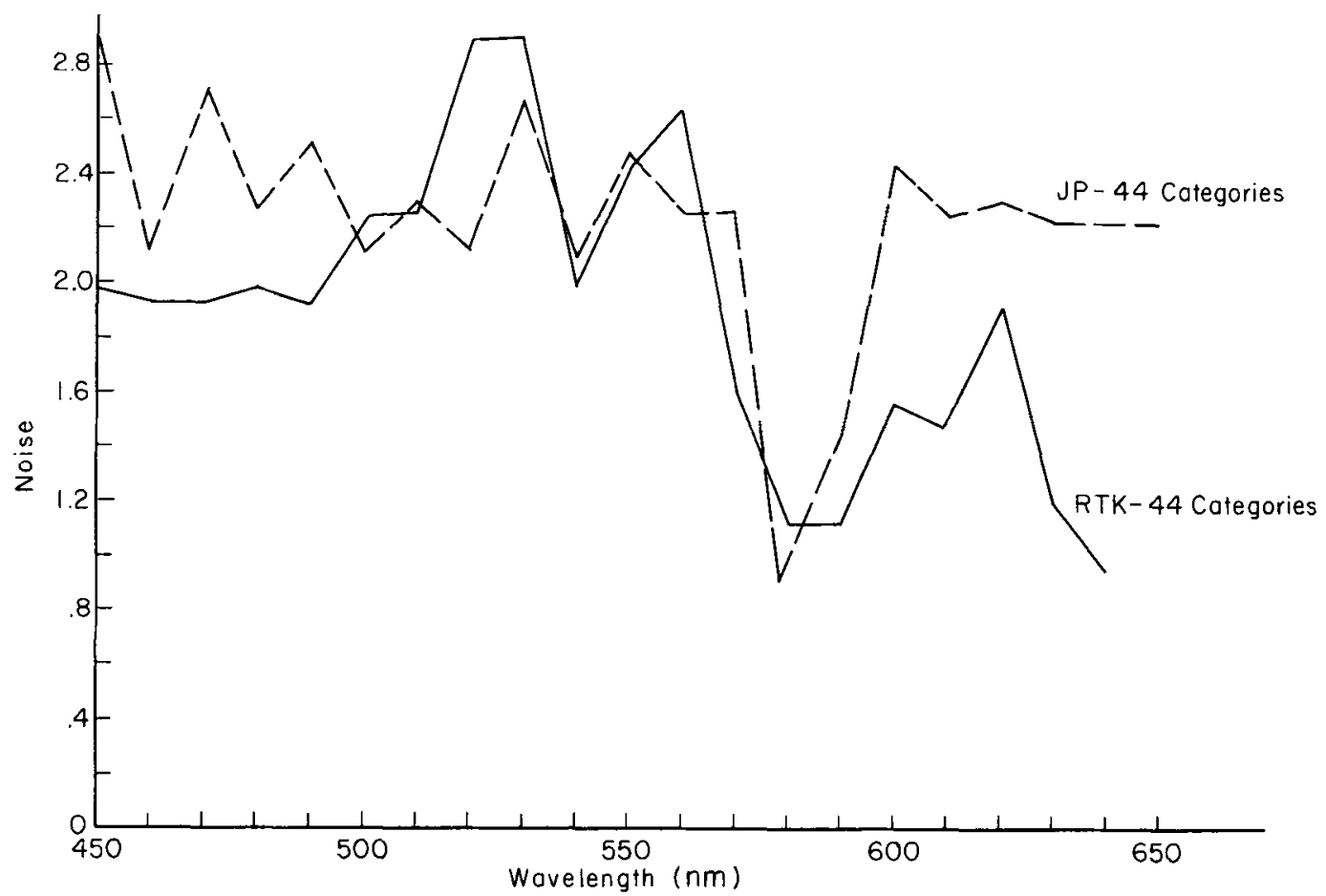

Fig. 3. Noise introduced by the $S$ as inferred from the distribution of responses to stimuli at each wavelength.

\section{The Optimal Number of Response Categories}

It can be concluded that the 12-category system, used in much of our previous work, was not adequate to allow maximum transfer of information through the S. For the conditions of this experiment, we conclude that the use of a 12-category condition results in the loss of about 0.6 bit of information relative to what can be achieved with the use of 44 categories.

Since the 44-category procedure allows more information to be transmitted than when the 24 categories are used, and is very close to the maximum of the fitted curve, it might seem reasonable to recommend using the 44-category method in future research. However, other considerations suggest that a 28-category procedure might be very satisfactory. Miller (1956) cites many examples to show that the maximum number of categories of absolute judgment that a human $\mathrm{S}$ can handle without error is normally in the range from six to eight. In terms of information transfer, this corresponds closely to the 2.6 bits that we have found in this study. Examination of the curve fitted to our experimental data suggests that the use of 28 categories would permit information transfer within $3 \%$ of maximum (2.49 bits). Unfortunately, we did not examine this condition experimentally, and it is not easily deducible by calculation from the 44-ategory condition. If employed, a 28-category condition would utilize seven steps of judgment between each of the adjacent unique hues, which seems a rational choice in view of the evidence cited by Miller. (To use a 28-category condition, a value of four would be assigned to the primary response, leading to the following response categories in the range from $\mathrm{B}$ to $\mathrm{G}: \mathrm{B}, \mathrm{BG} 1, \mathrm{BG} 2, \mathrm{BG} 3, \mathrm{~GB} 2$, $\mathrm{GB} 1$, and G.)

Noise Introduced by the Subject as a Function of Wavelength

In Fig. 3, an additional analysis of the results has been carried out as a function of wavelength. In order to compute the number of bits of response information, the analysis for each wavelength must be considered as being separate from that for other wavelengths. But for a single wavelength there is no information in the stimulus, so that response variabilityupon which the calculation of information depends-in fact is an index of noise introduced by the S. A most striking feature of this analysis is the minimum near $580 \mathrm{~nm}$, in the vicinity of unique yellow. This implies a very high precision and stability of the relation of outputs of the red and green mechanisms of the eye, whose balance is usually associated with the sensation of yellow.

\section{Comparison with Other Studies}

In a recent color-naming experiment, Beare and Siegel (1967) seem to imply that little is to be gained even by increasing the number of response categories from 6 to 11 . Their 6 categories included purple and orange plus the 4 that we have used; these were expanded to 11 with the addition of $B P, B G, Y G, Y O$, and OR, where the use of compounds did not permit the identification of a dominant component. These authors plot the raw curves for all 11 categories. The display of so many functions produces a confused picture which does not clearly reveal the relation between hue and wavelength. It was for this very reason that we had developed the point value system for reducing the display of response categories from 12 to 4 . Beare and Siegel also seem to place a high value on curves that reach $100 \%$, as many of theirs do for their six-category condition. This condition produces a range of $60 \mathrm{~nm}$ over which the name "Green" is always given and is thus plotted as $100 \%$. But stimuli over this range surely do not all appear exactly alike to the $S$ and it would seem that information about stimulus-response relations surely is being lost.

The main conclusion that Beare and Siegel draw from their study-with which we concur-is that even slight changes in the available response categories lead to substantial changes in response patterns; in particular when more categories are added, this changes the way that the original categories are used. But they make no computations and draw no conclusions about which type and/or number of categories seem optimal for the description of spectral colors.

The maximum information transmission in the present 
study occurred when the $\mathrm{S}$ was permitted use of 44 categories and produced the result close to 2.6 bits. This is considerably less than a mean value of 3.66 bits reported by Chapanis and Halsey (1956). Several reasons might account for this discrepancy. In their study Chapanis and Halsey did not gather data for subsequent analysis until Ss had reached a steady-state level of performance; this tequired as many as 550 trials in some cases. This prior training may produce a learned association between color names and stimuli which serves to increase transmission. Also, in the experiment reported here the stimuli were presented for only $250 \mathrm{msec}$ while the Ss in the Chapanis and Halsey experiment were permitted to view the stimuli continuously until a judgment was made.

In our experiment, the figure of about 2.6 bits of information transmission was derived from the use of 21 stimuli which were often "confused" with one another, in the sense that the same response category was used to "identify" more than one stimulus. It is tempting to conclude from this that it would be possible to select seven spectral stimuli that would never be confused with one another; such a total lack of confusion would imply complete transmission of the 2.6 bits of information in the stimulus. But Chapanis and Halsey continue to report "errors" for the smallest set of such carefully-chosen stimuli that they used (10), and one cannot be convinced that errors would have been eliminated completely with the use of an optimal seven. In practical situations, where correct recognition is often a life-and-death matter, more than six stimuli are virtually never used and the number used is usually much less than this. [See Halsey $(1959 \mathrm{a}, \mathrm{b})$ and Das (1966) for a discussion of some of these problems and for references to the signal-light literature.]

It should be emphasized, in conclusion, that our method does not presume the "correctness" of any response; indeed, the fact that the $S$ introduces noise in transmission information about a stimulus clearly implies that if stimulus categories are spaced closely enough, there is no "correct" response. The concept of "being correct" seems to us to have little meaning in fundamental comparisons of the relations between physical stimuli and the sensations that they produce.

\section{REFERENCES}

BEARE, A. C. Color-name as a function of wavelength. American Journal of Psychology, 1963, 76, 248-256.

BEARE, A. C., \& SIEGEL, M. H. Color name as a function of wavelength and instruction. Perception \& Psychophysics, 1967, 2, 521-527.

BOYNTON, R. M., \& GORDON, J. Bezold-Brucke hue shift measured by color-naming technique. Journal of the Optical Society of America, $1965,55,78-86$.

BOYNTON, R. M., SCHAFER, W., \& NEUN, M. E. Hue-wavelength relation measured by color-naming method for three retinal locations. Science, 1964, 146, 666-668.

CHAPANIS, A., \& HALSEY, R. M. Absolute judgments of spectrum colors. Journal of Psychology, 1956, 42, 99-103.

DAS, S, R. Recognition of signal colors by a different set of color names. Joumal of the Optical Society of America, 1966, 56, 789-794.

DIMMICK, F. L., \& HUBBARD, M. R. The spectral location of psychologically unique red. American Journal of Psychology, 1939, $52,348-353$.
DIMMICK, F. L., \& HUBBARD, M. R. The spectral location of psychologically unique yellow, green and blue. American Journal of Psychology, 1939, 52, 242-254.

GRAHAM, C. H. Discriminations that depend on wavelength. In C. H. Graham (Ed.), Vision and visual perception. New York: John Wiley and Sons, 1965.

HALSEY, R. M. Identification of signal lights: I. Blue, green, white and purple. Journal of the Optical Society of America, 1959, 49, 45-55.

HERING, E. Outlines of a theory of the light sense. Translated by L. M. Hurvich and D. Jameson. Cambridge: Harvard University Press, 1964.

JAMESON, D., \& HURVICH, L. Perceived color and its dependence on focal, surrounding and preceding stimulus variables. Journal of the Optical Society of America, 1959, 49, 890-898.

MILLER, G. A. The magical number seven, plus or minus two: Some limits on our capacity for processing information. Psychological Review, 1956, 63, 81-97.

SHANNON, C. E. A mathematical theory of communication. Bell System Technological Journal, 1948, 27, 379-423, 623-656.

STERNHEIM, C. E., \& BOYNTON, R. M. Uniqueness of perceived hues investigated with a continuous judgmental technique. Journal of Experimental Psychology, 1966, 72, 770-776.

THOMSON, L. C. Sensations aroused by monochromatic stimuli and their prediction. Optica Acta, 1954, 1, 93-102.

\section{NOTES}

1. These experiments were conducted in the Center for Visual Science at the University of Rochester under Grants NB-00624, Public Health Service, and GZ-414, National Science Foundation. John Parker held an R. Samuel McLaughlin Foundation Fellowship at the time the research was performed.

2. Address: Center for Visual Science, University of Rochester, Rochester, N.Y. 14627.

3. Now at the University of Toronto, Toronto, Ontario, Canada.

4. When each stimulus is presented an equal number of times (as in the experiment), the number of bits of information in the stimulus depends soleiy upon the number of possible stimuli. Nevertheless, the amount of information transmitted by the $\mathrm{S}$ could well depend upon how these stimuli are spaced along the spectrum. The number of bits of information in the stimulus first of all must be greater than what the $\mathrm{S}$ can transmit, in order for the latter to be measured. By choosing 21 stimuli, corresponding to 4.6 bits of information, we were working well beyond such a limit while staying within a practical range. Many investigators have used a $10-\mathrm{nm}$ spacing of stimuli, which leads from 20 to 30 stimuli, depending upon the range covered; this seems to have been deemed adequate for obtaining information-in the loose sense-about what a $S$ sees or can discriminate with respect to wavelength. But there is no profound rationale for using equal spacing on a wavelength, frequency, or other basis related solely to physical measurement. But unequal spacing can lead, in extreme cases to absurdities. Suppose, for example, that 19 of 21 stimuli were crowded between 630 and $640 \mathrm{~nm}$, with the other two at 450 and $550 \mathrm{~nm}$. Information transmission through the $S$ would doubtless be very low, since almost every stimulus would produce response distributions, however measured, that would overlap almost completely with those elicited by all but two of the others. We decided to space the stimuli an equal number of just-noticeable-differences apart using data from Graham (1965, p. 351). It is our opinion that the results would not differ in any important way had the usual even spacing with wavelength been employed.

5. The authors wish to thank Mr. Eugene Trabka, of the Eastman Kodak Research Laboratories, Rochester, New York, for his critical reading of the manuscript.

(Accepted for publication November 18, 1968.) 